BOLETIN DE LA SOCIEDAD GEOLOGICA MEXICANA

V. LIII, (2000) 84-92

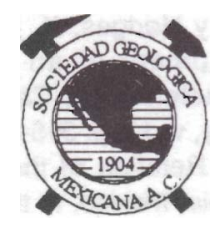

http://www.igeofcu.unam.mx/sgm/

\title{
Avances en el conocimiento de la Faja Volcánica Transmexicana durante la última década
}

\author{
Luca Ferrari \\ Instituto de Geología, UNAM, Apdo. Postal 70-296, Cd. Universitaria, \\ 04510 México D.F., luca@geologia.unam.mx
}

\begin{abstract}
Resumen
La Faja Volcánica Transmexicana (FVTM), el arco volcánico construido sobre la margen meridional de la Placa Norteamericana, presenta características peculiares tal como una gran variabilidad del estilo volcánico y de la composición química de sus productos, la oblicuidad de su parte central y oriental con la trinchera y la notable variación del ancho del arco. En esta reseña se sintetizan las principales contribuciones en campo geológico, geoquímico y geofísico sobre este arco volcánico según los artículos publicados durante la década de los noventa. Los mayores avances lo constituyen la reconstrucción de la estructura del sistema de subducción, el establecimiento de la edad y la evolución geológico-tectónica de la FVTM; la reconstrucción de la historia volcánica de los centros activos y la evaluación de su riesgo; y el esclarecimiento de la petrogénesis de los magmas. Sin embargo, a pesar de los avances, no se ha aclarado completamente el origen y la evolución de la FVTM. En el futuro, las investigaciones que pueden contribuir a solucionar los problemas de interpretación de la FVTM tendrán que enfocarse al conocimiento detallado de la geología del arco volcánico y de la estructura físico-química de la corteza y el manto por debajo de él.
\end{abstract}

\begin{abstract}
The Trans-Mexican Volcanic Belt (TMVB), the volcanic are built on the southern edge of the North America plate, presents several peculiar features such as large arc parallel variation in volcanic style and chemical composition, a slight obliquity with respect to the trench and a significant variation in the arc width. In this review I synthesize the main geologic, geophysics and geochemical contributions on this are based on papers published during the last decade. Significant advances in this respect were the reconstruction of the structure of the subduction system; the establishment of the age and geologic evolution of the TMVB; the reconstruction of the volcanic history of the active centers, the volcanic hazard assessment; and the deeper insight into the petrogenesis of the magmas. Despite these advances, the origin and the evolution of the TMVB is not completely understood yet. In the future, detailed geologic and tectonic studies of the arc and geophysical and geochemical investigations on the geometry and nature of the crust and underlying mantle may contribute to solve these problems.
\end{abstract}




\section{INTRODUCCiÓN}

La Faja Volcánica Transmexicana (FVTM) es el arco volcánico que se desarrolla sobre la margen sudoccidental de la Placa de Norteamérica como resultado de la subducción de las Placas Rivera y Cocos a lo largo de la trinchera de Acapulco (Figura 1). Los estudios sobre la FVTM comenzaron de manera puntual en la década de los sesenta. Una muestra representativa de los conocimientos obtenidos en aquella etapa la presenta Mooser (1972) quien también propone el primer modelo de la estructura del arco. En la década siguiente se realizaron los primeros trabajos sistemáticos que culminaron con la importante síntesis regional de Demant (1981). 'En los ochenta se llevaron a cabo trabajos volcanológicos y geoquímicos que aportaron conocimientos significativos sobre la mayoría de los grandes aparatos y campos volcánicos cuaternarios y se publicaron los primeros modelos sobre la tectónica de la parte occidental de la FVTM. El estado del arte para finales de aquella década fue resumido por Verma (1987) en ocasión de la publicación de un volumen especial de la revista Geofísica Internacional dedicado a la FVTM. En el presente trabajo se pretende sintetizar las contribuciones principales publicadas desde aquel entonces, así como señalar los problemas que quedan por resolver.

\section{CARACTERÍSTICAS DISTINTIVAS DE LA FVTM}

La FVTM constituye unos de los arcos volcánicos más complejos y variados de la región circum-Pacífica. Entre sus rasgos peculiares se pueden citar: a) la gran variabilidad del estilo volcánico y composición química a lo largo del arco; b) la presencia de un volcanismo alcalino de tipo intraplaca (asimilado a los Ocean Island Basalt) espacialmente asociado al volcanismo dominante relacionado con la subducción; c) la oblicuidad de su parte central y oriental con la trinchera; y d) la notable variación del ancho del arco (Figura 1).

a) La FVTM se suele dividir en tres sectores: occidental, central y oriental (Figura 2) que presentan diferencias significativas en lo que respecta al tipo de volcanismo y su composición química. En el sector occidental, limitado al este por el ritt de Colima, el arco volcánico se sobrepone a la parte norte del bloque Jalisco (BJ) y al límite meridional de la Sierra Madre Occidental (SMO). El volcanismo emplazado en el límite sur de la SMO es claramente bimodal, formado tanto por estratovolcanes y complejos de domos dacíticoriolíticos como por centros monogenéticos basálticos. En cambio, el volcanismo emplazado dentro del BJ es casi exclusivamente monogenético y de composición basáltico-andesítica.

En el sector central, entre el rift de Colima y el sistema de fallas Taxco-Querétaro (Figura 2), se desa-rrolla el campo volcánico Michoacán-Guanajuato, formado por centros monogenéticos y pequeños volcanes escudo de composición basáltica o basálticoandesítica. Los estratovolcanes están completamente ausentes, si se exceptúa el caso del Cerro Tancítaro.

En el sector oriental, al oriente del sistema TaxcoQuerétaro, la mayor parte del volcanismo está emplazado en grandes estratovolcanes, calderas y complejos de domos de composición de andesítica a riolítica alineados a lo largo de estructuras corticales, mientras que los volcanes monogenéticos de composición basáltica representan sólo una pequeña fracción del volumen de magma emplazado. En este sector el arco volcánico se emplaza sobre una corteza de edad precámbrica presumiblemente de tipo cratónico (Oaxaquia, Ortega-Gutiérrez et al., 1996).

b) Variedades de rocas volcánicas alcalinas han sido emplazadas a lo largo de toda la FVTM. Sólo una parte de las rocas alcalinas tiene afinidad intraplaca y puede interpretarse como basaltos de isla oceánica (OIB) derivados de un manto no contaminado por la subducción. Lavas OIB se han emplazado desde el Mioceno al Presente a lo largo de toda la FVTM pero más frecuentemente en el sector occidental (Tabla 1).

c) La FVTM no se encuentra a una distancia constante de la trinchera de Acapulco. Mientras que en el sector occidental el frente volcánico es paralelo a la trinchera, en el sector central y oriental tiende a alejarse de ella. Como resultado el frente volcánico tiene un ángulo de aproximadamente $15^{\circ}$ con respecto a la trinchera.

d) La tasa de emplazamiento volcánico y el área cubierta por el volcanismo Plio-Cuaternario no son homogéneas en la FVTM. El arco volcánico PlioCuaternario tiene un ancho variable entre un máximo de $200 \mathrm{~km}$ en la región del Campo Volcánico Michoacán-Guanajuato y un mínimo de $65 \mathrm{~km}$ en las regiones de Chapala y Morelia (Figura 1), que corresponden también a las áreas con menor aporte magmático del arco.

A diferencia de otros arcos volcánicos los rasgos peculiares descritos arriba no se relacionan directamente con la edad y la estructura térmica de las placas en subducción. La litósfera oceánica en 


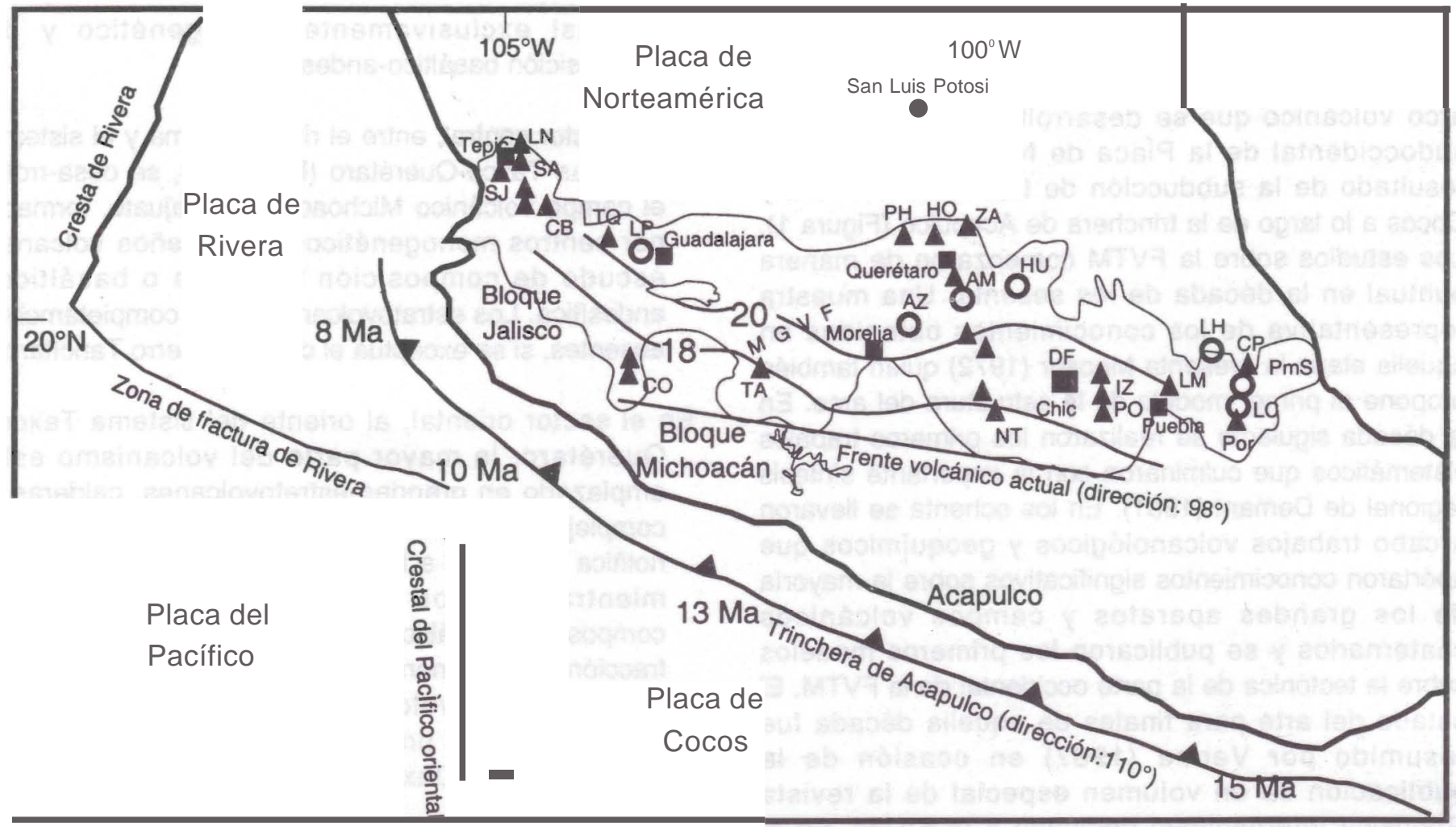

Figura 1. Entorno tectónico y principales centros volcánicos de la Faja Volcánica Transmexicana. las edades en la trinchera se refieren a la placa que entra en la zona de subducción. Las líneas interrumpidas representan la posición de las isócronas de la placa subducida, cuyas edades se indican (basado en Kostoglodov y Bandy, 1995, corregido por la inclinación del plano de Benioff de acuerdo a la estimación de Pardo y Suárez, 1995). Ios triángulos indican los principales estrato-volcanes; los mencionados en el texto son: IN =Las Navajas; SA =sangangüey; SJ =San Juan; $\mathrm{CB}=$ Ceboruco; $\mathrm{TQ}=$ Tequila; $\mathrm{CO}=$ Volcán de Colima; TA =Tancítaro; PH = Palo Huérfano; HO =La Joya; ZA =El zamorano; NT = Nevado de Toruca; $I Z=$ Iztaccíhuatl; PO =Popocatépetl; IM =la Malinche; $\mathrm{CP}=$ Cofre de Perote; Por =Pico de Orizaba. Ios círculos indican las

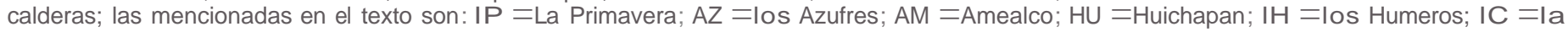
Cumbre. Ios principales campos volcánicos mencionados en el texto son: MGVF = campo volcánico Michoacán-Guanajuato; Chic $=$ Sierra Chichinautzin; PmS =Chiconquiaco-Palma Sola.

subducción en la trinchera tiene edades variables entre 8 y 15 Ma pero por debajo del arco volcánico PlioCuaternario se encuentra una placa de edad uniforme de entre 18 y 20 Ma (Bandy y Kostoglodov, 1995) (Figura 1).

\section{AVANCES EN LA ÚLTIMA DÉCADA}

\section{Edad y evolución geológica de la FVTM}

La edad de la FVTM ha sido un tema controversia!. En el pasado se habían sugerido fechas variables desde Cuaternario (Demant, 1978; 1981), Plioceno tardío (Cantagrel y Robin, 1979), Plioceno temprano (Nixon et al., 1987) hasta Oligoceno (Mooser, 1972). Sin embargo, en la última década, la cartografia geológica apoyada en un gran número de nuevas edades isotópicas, ha llegado a esclarecer la evolución espacio-temporal del volcanismo en México central. La recopilación.y el análisis de varios centenares de edades ha permitido visualizar la reorientación progresiva del volcanismo continental desde la Sierra Madre Occidental a la FVTM, indicando que un arco volcánico con la orientación y las características químicas de esta última existía ya desde el Mioceno superior (Ferrari et al., 1994a, y 1999a) (Figura 2). Desde entonces el frente volcánico ha migrado hacia la trinchera, sobre todo en la parte occidental y central de la FVTM (Ferrari et al., 1994a; Delgado et al., 1995).

Estos estudios han permitido además reconocer que el volcanismo de la FVTM se caracteriza por pulsos de mayor intensidad separados por periodos de actividad reducida (Figura 3). El primer pulso tuvo lugar al inicio del Mioceno tardío (10-9 Ma), cuando la FVTM se caracteriza por el emplazamiento de grandes mesetas andesítico-basálticas en el sector occidental y central (Moore et al., 1994; Ferrari et al., 1994b, 1999c; Righter et al., 1995) y de estratovolcanes andesíticos como el Zamorano (Carrasco-Núñez et al., 1989) Palo Huérfano (Pérez-Venzor et al., 1997), La Joya (Valdez-Moreno et al. 1996) y Cerro Grande (Carrasco-Núñez et al., 1997) en el oriente. Posteriormente a los $8 \mathrm{Ma}$, se observa 


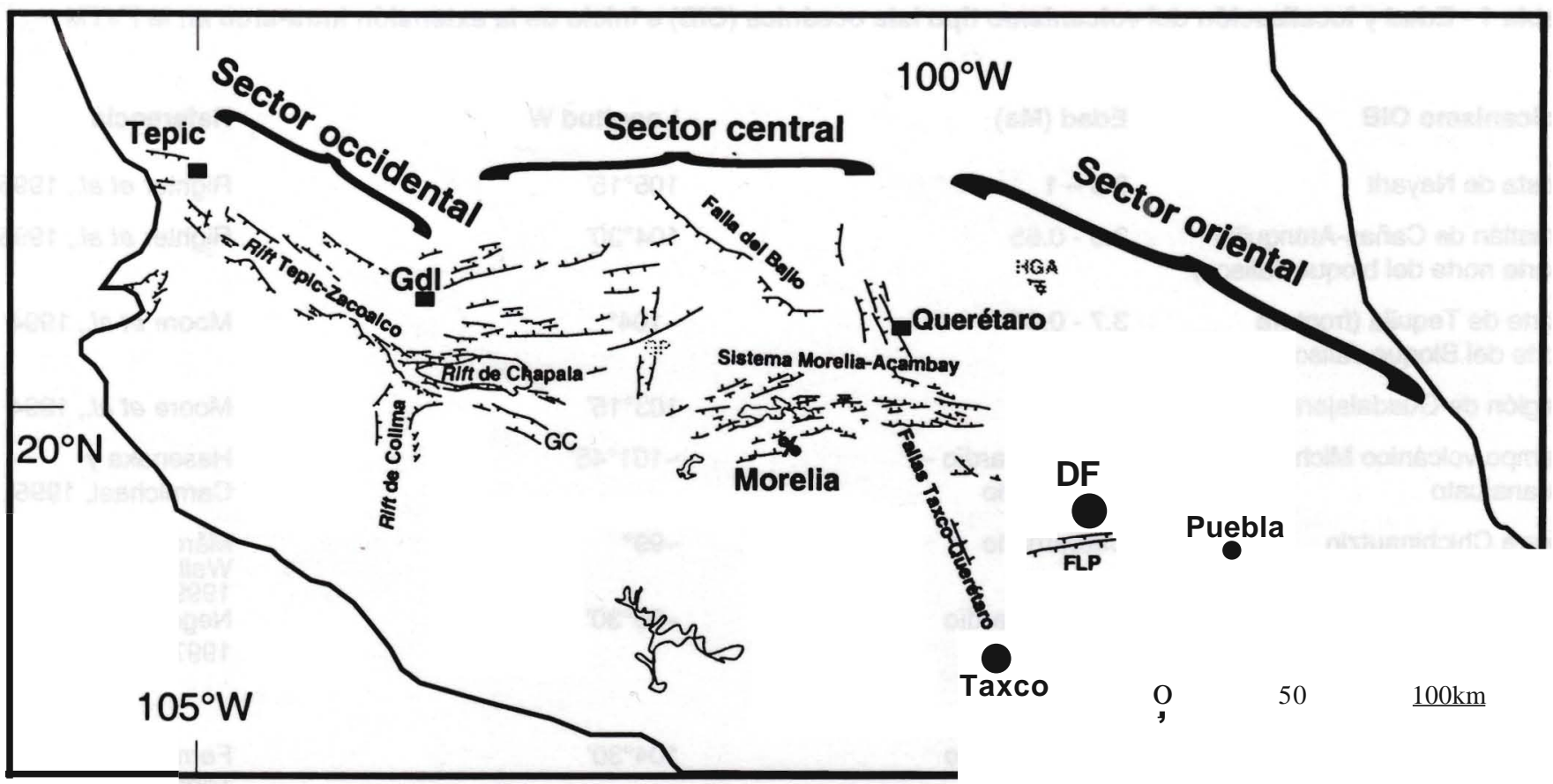

Figura 2. Principales sistemas de fallas con edades inferiores a los 10 Ma que afectan a la Faja Volcánica Transmexicana (basados en Ferrari y Rosas-Elguera, 1999a; Ferrari et al., 1999c; Alaniz-Á1varez et al., 1998). GC = Graben de Cotija; GP = Graben de Penjamillo; HGA = Semigraben de Aljibes; FLP =Falla de La Pera. Nótese la ausencia de fallamiento en la parte oriental de la FVTM.

una disminución del volcanismo a lo largo de toda la FVTM. En el Plioceno inferior (5-3 Ma) el volcanismo se vuelve marcadamente bimodal: complejos de domos riolíticos se emplazaron entre Tepic y Guadalajara (Gilbert et al., 1985; Righter et al., 1995; Ferrari et al., 1997, 1999b) Y en el sector central de la FVTM (Pasquaré et al., 1991), donde además se formaron las calderas de Los Azufres, Amealco y Huichapan (Ferrari et al., 1991; Aguirre-Díaz, 1997; Aguirre-Díaz et al., 1997). En el sector occidental el comienzo de este volcanismo pliocénico está marcado por el emplazamiento de basaltos alcalinos entre Tepic y Guadalajara (Moore et al., 1994; Righter et al., 1995) y en la parte norte del rift de Colima (Allan, 1986). Un último pulso volcánico parece caracterizar el Plioceno tardío-Cuaternario (Figura 3), cuando la composición dominante de los productos se vuelve otra vez intermedio-máfica.

\section{Marco geodinámico y tectónico}

La relocalización precisa de los sismos de subducción bajo México central junto con estudios magneto-telúricos han permitido reconstruir la geometría de las placas en subducción, evidenciando que el frente volcánico Cuaternario se encuentra en correspondencia de los $80-100 \mathrm{~km}$ de profundidad del plano de Beniotf. De acuerdo a estos datos el ángulo de $-15^{\circ}$ que guarda el frente volcánico de la FVTM respecto a la trinchera de Acapulco resultarra ser una consecuencia de la variación de la inclinación de la placa en subducción. En particular, el plano de Benioff que define la placa de Rivera por debajo del bloque Jalisco tendrra un ángulo de $45-50^{\circ}$ (Pardo y Suárez, 1993; 1995), se volverra progresivamente más somero en correspondencia de Michoacán hasta volverse subhorizontal en Guerrero y Oaxaca (Pardo y Suárez, 1995; Arzate et al., 1995). El ángulo aumentarra otra vez en correspondencia de la región rstmica y centroamericana (Pardo y Suárez, 1995). Sin embargo, los sismos son casi ausentes por debajo de la FVTM, lo que deja mucha incertidumbre sobre la geometrra de la placa en subducción en esta región. El análisis de los eventos recopilados en el catálogo sísmico del Servicio Sismológico Nacional (R. Zúñiga, comunicación personal) sugiere que la placa podrra hundirse con alto ángulo en correspondencia de la FVTM.

Datos gravimétricos (Urrutia-Fucugauchi y Flores-Ruiz, 1996) indican que el espesor cortical de la placa superior es máximo en la parte oriental de la FVTM, donde llega a los $50 \mathrm{~km}$. En esta región se infiere la existencia de un núcleo de basamento Precámbrico (Ortega-Gutiérrez et al., 1995). Hacia el occidente el espesor disminuye considerablemente, hasta llegar a $28 \mathrm{~km}$ en Arteaga (Pérez-Garcra y Urrutia-Fucugauchi, 1997), al suroeste del campo volcánico Michoacán-Guanajuato. 
Tabla 1 - Edad Y localización del volcanlsmo tipo Isla oceánica (018) e Inicio de la extensión Intra-arco en la FVTM

Volcanlsmo 018

Costa de Nayarit

Amatlán de Cañas·Atenguillo (parte norte del bloque Jalisco)

Norte de Tequila (frontera

norte del Bloque Jalisco)

Región de Guadalajara

Campo volcánico Miehoacán-

Guanajuato

Sierra Chichinautzin

Chiconquiaeo-Palma Sola

Inicio de la extensión

Este de Tepie

Región del Ceborueo

Lago de Chapala

Lago de Cuitzeo

La deformación en la placa superior se concentra principalmente a lo largo de sector occidental y central de la FVTM. Una tectónica transcurrente y/o transtensional caracteriza estas regiones en el Mioceno medio y tardío (Ferrari, 1995; Ferrari et al., 1994a, 1994c, 1997, 1999c). Sin embargo, desde finales del Mioceno, ha actuado una extensión casi perpendicular al arco volcánico. Suter et al. (1992 y 1995a y b) han documentado en detalle la extensión intra-arco reciente y activa en la parte central de la FVTM, calculando tasas de extensión entre 0.7 y 0.4 mm/año. De manera similar, en la parte occidental de la FVTM, se ha reconocido una extensión perpendicular al arco con tasas de extensión inferiores al mm/año activa desde el Mioceno tardío (Barrier et al., 1990; Nieto-Obregón et al., 1992; Ferrari et al., 1994c; Suárez et al., 1994; Ferrari y Rosas, 1999a). La ausencia de fallamiento transcurrente derecho en el Pifo-Cuaternario a lo largo del rift Tepic-Zacoalco y la baja tasa de deformación de estos sistemas de fallas han puesto en duda el modelo de rifting hacia el noroeste del bloque Jalisco por efecto de un "brinco" hacia el este de la cresta de Pacifico oriental (Luhr et al., 1985; Allan et al., 1991), que había dominado en. la literatura en la década de los ochenta. E[1 la actualidad, se considera más probable que la
LongitudW

$105^{\circ} 15^{\prime}$

$104^{\circ} 30^{\prime}$

$-104^{\circ}$

$103^{\circ} 15^{\prime}$

$-101^{\circ} 45^{\prime}$

$-99^{\circ}$

$-96^{\circ} 30^{\prime}$

$104^{\circ} 30^{\prime}$

$104^{\circ} 30^{\prime}$
Referencia

Righter et al., 1995

Righter et al., 1995

Moore et al., 1994

Moore et al., 1994

Hasenaka y

Márquez et al., 1999; 1999

Negendank et al., 1997

Ferrari et al., 1997, 1999

Ferrari et al., 1997, 1999

Rosas-Elguera y 1998

Israde-Alcántara, 1997.
Carmichael,1995 Wallace y Carmichael, Urrutia-Fucugauchi,

tectónica extensional del la FVTM occidental estuvo relacionada con la reactivación de las fronteras del bloque Jalisco inducida por las fuerzas aplicadas en los límites de placas y, en particular, por la diferente geometría de las placas de Rivera y Cocos subducidas bajo esta parte de México (Bandy et al., 1995; Rosas et al., 1996).

Un modelo que relaciona la tectónica con el estilo del volcanismo ha sido recientemente propuesto por Alaniz-Alvarez et al. (1998, 1999), quienes, partiendo del modelo teórico de Takada (1994), propusieron que los centros poligenéticos mayores de la FVTM están alineados a lo largo de las estructuras transversales al arco debido a que estas últimas tienen una baja tasa de deformación. Este modelo fue cuestionado por Suter (1999), Contreras y Gómez Tuena (1999) y Siebe et al. (1999) quienes pusieron en duda la aplicabilidad del modelo de Takada a la FVTM y la simplificación de la tectónica de la región propuesta por Alaniz-Álvarez et al. (1998). Sin embargo, considerando que la deformación puede ser liberada por el magmatismo (Alaniz-Álvarez et al., 1999) y que no se cuestionó que la velocidad de extensión en zonas transversales al arco debe ser muy pequeña, se puede decir que el 


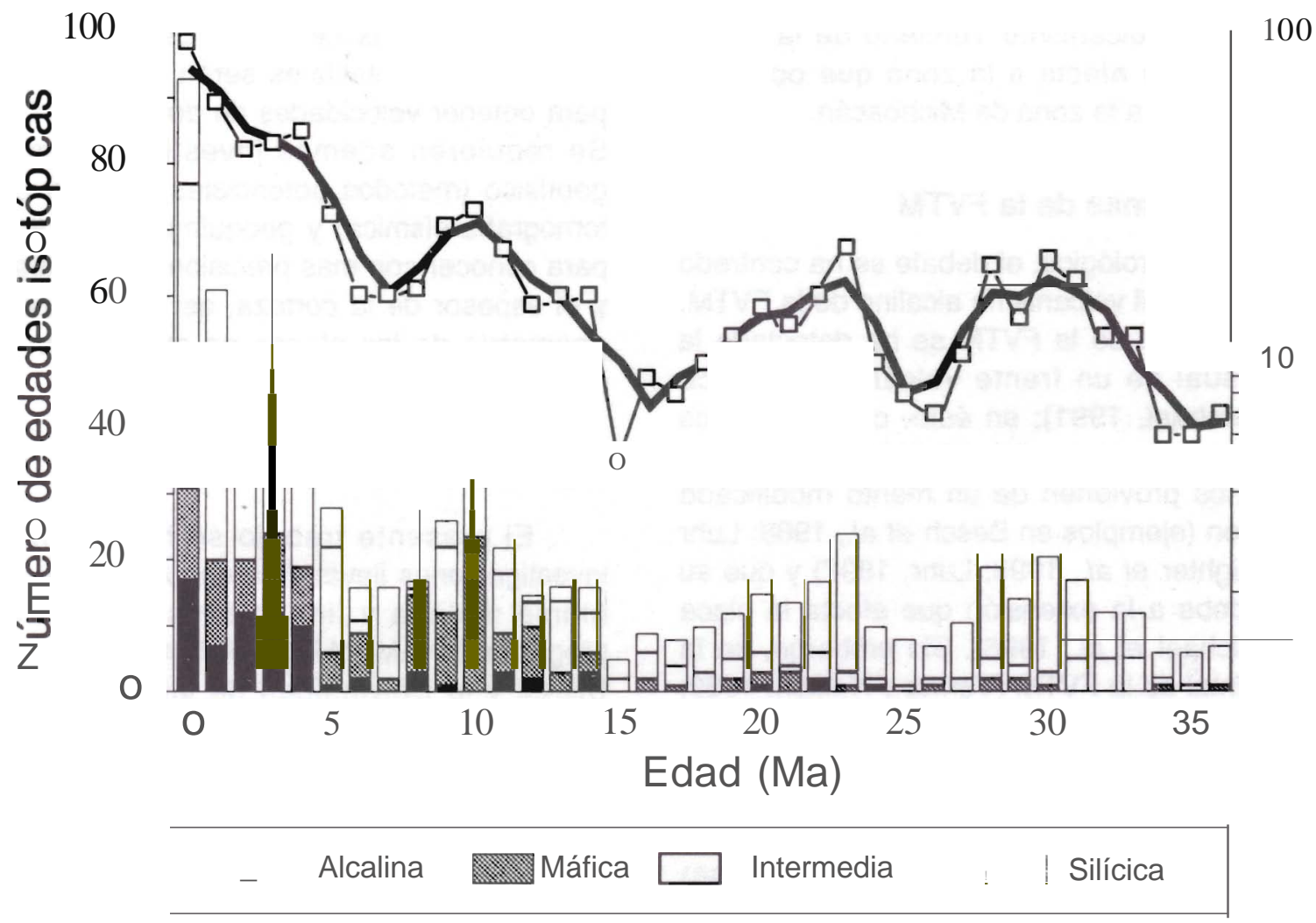

Figura 3. Distribución en valores absolutos (escala en la izquierda) y logarítmicos (escala en la derecha) de $n 1$ edades radiométricas para México central por intervalos de $1 \mathrm{Ma}$. La distribución multimodal sugiere que el volcanismo haya ocurrido en pulsos. La línea gruesa se obtuvo promediando dos valores adyacentes. Tomado de Ferrari et al. (1999a).

modelo de Alaniz-Álvarez y colaboradores (1998) explica la distribución peculiar del volcanismo monogenético y poligenético de la FVTM.

\section{Geología regional y local}

Muchos estudios volcanológicos y geológicos se han concentrado sobre los centros volcánicos que presentan mayor riesgo para la población. En este campo, la contribución mayor de la década ha sido probablemente la de reconocer que la mayoría de los grandes estratovolcanes andesítico-dacíticos de la FVTM tuvieron uno o más colapsos sectoriales durante el Holoceno. Depósitos de avalancha de escombros relacionados con estos fenómenos cataclísmicos han sido detectados en los volcanes Colima (Luhr y Prestegaard, 1988; Stoopes y Sheridan, 1992), Jocotitlán (Siebe et al., 1992), Nevado de Toluca (Macías et al., 1997), Popocatépetl (Robin y Boudal, 1987; Siebe et al., 1995) y Pico de Orizaba (CarrascoNúñez et al., 1993; Hoskuldsson y Robin, 1993, Carrasco-Núñez y Gómez-Tuena, 1997).

Además, han sido publicados mapas geológicos con diferente grado de detalle de los volcanes Colima
(Rodríguez-Elizarrarás, 1995), Las Navajas (Nelson y Hegre, 1990), Nevado de Toluca (Macías et al., 1997); Iztaccíhuatl (Nixon, 1989) y Pico de Orizaba (CarrascoNúñez y Ban, 1994). Sin embargo, volcanes importantes como el Cofre de Perote, el Volcán San Juan, La Malinche y, en parte, el mismo Popocatépetl carecen de un mapa geológico completo. También se han realizado estudios geológicos y geocronométricos detallados de las calderas y complejos dómicos de edad Plioceno de la parte central de la FVTM (Amealco, Huichapan, Los Agustinos, etc.) (AguirreDíaz, 1997; Aguirre-Díaz et al., 1997). Estudios similares de otros complejos caldéricos, como Los Azufres (Ferrari et al., 1991; Pradal y Robin, 1994; Campos-Enríquez y Garduño-Monroy, 1995) y Zitácuaro (Capra et al., 1997), han mostrado que éstos se caracterizan por una historia más larga, iniciada posiblemente en el Mioceno y caracterizada por varias reactivaciones hasta el Cuaternario.

La geología regional de la parte central y occidental de la FVTM ha sido sintetizada en los trabajos cartograficos de Pasquaré et al. (1991), Ferrari et al. (1999b) y Rosas-Elguera et al. (1997). Estos trabajos han contribuido a establecer los límites entre 
Norteamérica y los bloques Jalisco y Michoacán y han demostrado que el volcanismo Terciario de la Sierra Madre Occidental no afecta a la zona que ocupa el bloque Jalisco pero sf a la zona de Michoacán.

\section{Origen de los magmas de la FVTM}

En el campo petrológico, el debate se ha centrado sobre el significado del volcanismo alcalino de la FVTM. En la parte occidental de la FVTM se ha detectado la existencia inusual de un frente volcánico potásico (Lange y Carmichael, 1991); en éste, como en otros casos, los patrones de elementos traza indican que los magmas alcalinos provienen de un manto modificado por la subducción (ejemplos en Besch et al., 1988; Luhr et al., 1989; Righter et al., 1995; Luhr, 1997) y que su ocurrencia se debe a la extensión que afecta la placa superior (Carmichael et al., 1996). Sin embargo, en la parte noroccidental de la FVTM (Verma y Nelson, 1989; Wallace et al., 1992; Righter y Carmichael, 1992), en el extremo oriental (Palma Sola, Negendank et al., 1987) asf como en la Sierra Chichinautzin, ubicada en el frente volcánico de la parte central (Márquez et al., 1999; Verma, 1999; Wallace y Carmichael, 1999) existen lavas que se parecen a los basaltos de isla oceá-nica (OIB), lo que implicarfa un manto no contaminado por la subducción. Basándose sobretodo en estas ocurrencias, Márquez et al.(1999) propusieron un modelo provocativo en el cual los magmas OIB serfan la expresión de una pluma del manto que estarfa moviéndose de oeste a este desde el Mioceno tardfo, por debajo de la FVTM. El problema principal de este modelo lo representa la evolución temporal del fallamiento y del volcanismo OIB en la FVTM, que no muestra la propagación predicha por el modelo (Tabla 1) y el volumen de las lavas OIB que resulta ser muy por debajo a lo esperado si existiera una pluma del manto (Ferrari y Rosas-Elguera, 1999b). Un modelo alternativo ha sido propuesto en forma general por Luhr (1997), quien su-giere que la ocurrencia de lavas OIB pueda relacionarse con un flujo de material no contaminado desde la zona de retroarco inducido por la subducción.

\section{OPORTUNIDADES DE INVESTIGACIÓN FUTURAS}

En general, los problemas de interpretación de la FVTM derivan de la falta de un conocimiento detallado de la geologfa del arco volcánico y de la estructura ffsico-qufmica de la corteza y el manto por debajo de ella. Falta todavfa cartograffa geológica y estratigraffa sistemática que abarque toda la FVTM y que permita la estimación de las tasas de efusión volcánica por áreas en el tiempo y de las tasas de deformación de los diferentes sistemas de fallas. Estudios geodésicos que utilizan redes satelitales serán también fundamentales para obtener velocidades de deformación instantáneas. Se requieren además investigaciones de carácter geoffsico (métodos potenciales, sfsmica de refracción, tomograffa sfsmica) y geoqufmico (estudios isotópicos) para conocer con mas precisión la naturaleza geológica y el espesor de la corteza, asf como la extensión y la geometrfa de las placas en subducción y la composición del manto.

\section{AGRADECIMIENTOS}

El presente trabajo se ha beneficiado de las investigaciones llevadas a cabo por el autor durante la última década y financiadas por el CONACyT, el programa UNAM-PAPIIT, el Instituto de Geologfa de la UNAM y la Universidad de Milán, Italia. La revisión crftica de A. Nieto Samaniego, G. Carrasco-Nuñez y S. A. Alaniz estimuló una ampliación del manuscrito original que redundó en un trabajo más completo.

\section{REFERENCIAS BIBLIOGRÁFICAS}

Aguirre-Díaz, G., 1997, Volcanic stratigraphy of the Amealco Caldera and vicinity, central Mexican Volcanic Belt: Revista Mexicana de Ciencias Geológicas, v. 13, p. 10-51.

Aguirre-Díaz, G. J.; Nelson, S. A., Ferrari, L., y López-Martínez, M., 1997, Ignimbrites of the central Mexican Volcanic Belt-Amealco y Huichapan calderas, states of Querétaro and Hidalgo: Magmatism and tectonics of central and northwestern Mexico. A selection of the 1997 IAVCEI General Assembly Excursions. Instituto de Geología, UNAM, 1-39.

Alaniz-Álvarez. S.A.; Nieto-Samaniego, A.F., y Ferrari, L., 1998, Effect of strain rate in the distribution of monogenetic and polygenetic volcanism in the Transmexican Volcanic Belt. Geology, 26, p.591-594.

AJaniz-Álvarez S. A., Nieto-Samaníego A.F., y Ferrari L., 1999, Effect of the Strain Rate in the Distribution of Monogenetic and Polygenetíc Volcanism in the Transmexican Volcanic Belt. Reply to comments by M. Suter, Contreras y Gómez-Tuena; Siebe et al., Geology, 27, p. 573-575.

Allan J. F., Nelson S.A., Luhr J. F., Carmichael I.S.E., Wopat M. y Wallace P.J., 1991, Pliocene-recent rifting in SW Mexico and assocíated volcanism: an exotic terrain in the making: American Association of Petroleum Geologists, Memoir 47, p. 425-445.

Arzate. J. A.; Mareschal, M., y Livelybrooks, D., 1995, Electrical image of the subducting Cocos plate from magnetotelluric observations: Geology, 23, p. 703-706.

Bandy, W.L., Mortera-Gutierrez, CA, Urrutia-Fucugauchi, J., y Hilde, T.W.C., 1995, The subducted Rivera-Cocos plate boundary: where is it, what is it and what is its relationship to the Colima rift?: Geophysical Research Letters, 22, p. 3,075-3,078.

Barrier. E., Bourgois, J., y Michaud F., 1990, Le systeme de ríft actifs du point triple de Jalisco-vers un proto-golfe de Jalisco: C. R. Acad. 5ci. Paris, 310, p. 1,513-1,520.

Besch,T.H., Negendank, J.F'w., Emmermann, R., y Tobschall, H.J., 1989, Geochemical constraints of the origin of calcalkaline and alkaline magmas of the eastern transmexican volcanic belt: Geofisíca Internacional, 27, p. 641-663. 
Campos-Enríquez, O., Y Garduño-Monroy, V.H., 1995, Los Azufres silicic center (Mexico)-inference of caldera structural elements from gravity, aeromagnetic and geoelectric data: Journal of Volcanology and Geothermal research, 67, p. 123-152.

Cantagrel, J. M., Y Robin, C., 1979, K-Ar dating on Eastem Mexican volcanic rocks-Relations between the andesitic and the alkaline Provinces: Journal of Volcanological and Geothermal Research, v. 5, p. 99-114.

Capra, L.; Macías, J.L., y Garduño, V.H., 1997, The Zitácuaro Volcanic Complex, Michoacán, Mexico-magmatic and eruptive history of a resurgent caldera: Geofísica Intemacional, 36 , p. $161-180$.

Carmichael, I.S.E.; Lange, R.A., y Luhr, J.F., 1996, Quaternary minettes and associated volcanic rocks of Mascota, western Mexico-a consequence of plate extension above a subduction modified mantle wedge: Contributionsto Mineralogy and Petrology, 124, p. 302-333.

Carrasco-Núñez, G.; Milán, M., y'Verma, S.P., 1989, Geología del volcán El Zamorano, Estado de Querétaro: Universidad Nacional Autónoma de México: Instituto de Geología, Revista, v.8, p. 194-201.

Carrasco-Núñez, G., Vallance, J.w., y Rose, W.I., 1993, A voluminous avalanche-induced lahar from Citlaltépetl volcano, Mexicoimplications for hazard assessment: Journal of Volcanology and Geothermal Research, 59, p. 35-46.

Carrasco-Núñez, G., y Ban, M., 1994, Geologic map and structure sections of the Citlaltépetl volcano summit area, Mexico: Cartas Geológicas y Mineras 9. Instituto de Geología, UNAM.

Carrasco-Núñez, G. ; Gomez-Tuena, A., y Lozano, L., 1997, Geologic map of Cerro Grande volcano and surrounding area, Central Mexico: Geological Society of America Maps and Charts series, $\mathrm{MCH} 081,10 \mathrm{p}$

Delgado-Granados,.H.; Urrutia-Fucugauchi, J.; Hasenaka, T., y Ban, M., 1995, Southwestward volcanic migration in the western Trans-Mexican Volcanic Belt during the last 2 Ma: Geofisica Internacional, 34, p. 341-352.

Demant, A., 1978, Características del eje neovolcánico transmexicano y sus problemas de interpretación: Universidad Nacional Autonoma de México, Instituto de Geología, Revista, v. 2, p. 172-187.

Ferrari, L., 1995, Miocene shearing along the northern boundary of the Jalisco block and the opening of the southern Gulf of California: Geology, 23, p. $751-754$

Ferrari L. y Rosas-Elguera J., 1999a, Late Miocene to Quaternary extension at the northern boundary of the Jalisco block, western Mexico-the Tepic-Zacoalco rift revised: Geological Society America Special Paper \#334, Chapter 03, en prensa.

Ferrari L., y Rosas-Elguera, J., 1999b, Alkalic (OIB type) and calealkalic volcanism in the Mexican Volcanic Belt: a case for plume-relatad magmatism and propagating rifting at an active margin? Comment a un articulo de Marquez, A., Oyarzun, R., Doblas, M., Yerma, S. P., Geology, v. 27, p. 1,055-1,056.

Ferrari, L., Garduño, V.H., Pasquare, G., y Tibaldi, A., 1991, Geology of Los Azufres caldera, Mexico, and its relations with regional tectonics: Journal of Volcanology and Geothermermal Research, 47, p. 129-148.

Ferrari, L., Garduño, V.H., Pasquare, G., y Tibaldi, A., 1994a, Volcanic and tectonic evolution of Central Mexico-Oligocene to Present: Geofísica Internacional, 33, p. 91-105.

Ferrari, L., Garduño, V.H., Innocenti, F.; Manetti, P.; Pasquare, G., y Vaggelli, G., 1994b, A widespread mafic volcanic unit at the base of the Mexican Volcanic Belt between Guadalajara and Queretaro: Geofísica Internacional, 33, p. 107-124.

Ferrari, L., Pasquare, G., Venegas, S., Castillo, D., y Romero, F., 1994c, Regional tectonics of western Mexico and its implications for the northern boundary of the Jalisco Block: Geofísica Internacional, 33, p. 139-151-

Ferrari, L.; Nelson, SA, Rosas-Elguera, J, y Venegas-Salgado, S.,
1997, Tectonics and volcanism of the western Mexican Volcanic Belt: Magmatism and Tectonics of central and northwestern Mexico. A selection of the 1997 IAVCEI General Assembly Excursions. Instituto de Geología, UNAM, p. 85-129.

Ferrari L., López-Martínez M., Aguirre-Díaz G. y Carrasco-Nuñez G., 1999a, Space-time patterns of Cenozoic are volcanism in central Mexico-from the Sierra Madre Occidental to the Mexican Volcanic Belt: Geology, 27, p. 303-307

Ferrari L., Pasquaré G., Venegas S. y Romero F., 1999b, Geology of the westem Mexican Volcanic Belt and adjacent Sierra Madre Occidental and Jalisco block: Geological Society America Special Paper \#334, Chapter 04, en prensa.

Ferrari L., Conticelli S., Vaggelli C., Petrone C. y Manetti P., 1999c, Late Miocene mafic volcanism and intra-arc tectonics during the early development of the Trans-Mexican Volcanic Belt: Teetonophysics, en prensa.

Garcia-Perez F., y Urrutia-Fucugauchi J., 1997, Crustal structure of the Arteaga Complex, Michoacán, Southern Mexico, from gravity and magnetics: Geofísica Intemacional, 36, p. 235-244.

Gilbert C. M., G. Mahood y 1. S. E. Carmichael, 1985, Volcanic stratigraphy of the Guadalajara area, Mexico: Geofisica Intemacional, v. 24, p. 169-191.

Gómez-Tuena, A., y Carrasco-Nuñez, G., 2000, Cerro Grande volcano-the evolution of a Miocene stratocone in the early Trans-Mexican Belt: Teetonophysucs, en prensa.

Hasenaka T. y 1. S. E. Carmichael, 1985, A compilation of location, size and geomorphological parameters of volcanoes of the Michoacan-Guanajuato volcanic field, central Mexico: Geofisica Intemacional, v. 24, p. 5n-607.

Hasenaka T., 1994, Size, distribution and magma output rate for shield volcanoes of the Michoacan-Guanajuato volcanic field, central Mexico: Journal of Volcanology and Geothermal Research, 63, p. 13-31.

Hoskuldsson, A., y Robin, C., 1993, Late Pleistocene to Holocene eruptive activity of Pico de Orizaba, eastern Mexico: Bulletin of Volcanology, 55, p. 571-587.

Israde-Alcantara, 1., 1997, Neogene diatoms of Cuitzeo Lake, central Trans-Mexican Volcanic Belt and their relations with the volcano-tectonic evolution: Quaternary Intemational, v. 43144, p. 137-143.

Kostoglodov, V., y Bandy W., 1995, Seismotectonic constraints on the convergence rate between the Rivera and North American piates: Journal of Geophysical Research, v. 100, p. 17,9n17,989 .

Lange, R., y Carmichael, I.S.E., 1991, A potassic volcanic front in western Mexico-lamprophyric and related lavas of San Sebastian: Geological Society of America Bulletin, 103, p.928-940.

Luhr, J., 1997, Extensional tectonics and the diverse primitive volcanic rocks in the western Mexican Volcanic Belt: The Canadian Mineralogist, 35, p. 473-500.

Luhr, J., y Prestegaard, K., 1988, Caldera formation at Volcan Colima, Mexico, by a large Holocene volcanic debris avalanche: Joumal of Volcanology and Geothermal Research, 35, p. 335-348.

Luhr J. F., S. A. Nelson, J. F. Allan y 1. S. E. Carmichael, 1985, Active rifting in Southwestern Mexico-manifestations of an incipient eastward spreading-ridge jump: Geology, 13, p. 54-57.

Luhr, J.; Allan, J.; Carmichael, I.S.E., Nelson S.A., y Hasenaka T., 1989, Primitive calc-alkaline and alkaline 'rock type from the western Mexican Volcanic Belt: Journal of Geophysical Research, 94, p.4,515-4,530.

Macías, J.L.; García, P. A.; Arce, J.L.; Siebe, C.; Espíndola, J.M.; Komorowski, J.C., y Scott, K., 1997, Late Pleistocene-Holocene cataclysmic eruptio(1s at Nevado de Toluca and Jocotitlan volcanoes, Central Mexico, in Link, K.P., y Kowallis, B.J. (eds.), proterozoic to recent stratigraphy, tectonics and volcanology, UTAH, Nevada, southem Idaho and Central Mexico. Bringham 
Young University, Geology Studies, 42, part 1, p. 493-528.

Marquez, A., Oyarzun, R., Doblas, M., Verma, S. P., 1999, Alkalic (OIB type) and calc-alkalic volcanism in tile Mexican Volcanic Belt: a case for plume-related magmatism and propagating rifting at an active margin?: Geology, v. 27, p. 51-54.

Moare, G.; Marone, C.; Carmichael, I.S.E., y Renne, P., 1994, Basaltic volcanism and extension near the intersection of the Sierra Madre volcanic province and the Mexican Volcanic Belt: Geological Society of America Bulletin, v. 106, p. 383-394.

Meoser, F., 1972, The Mexican Volcanic Belt-Structure and tectonics: Geofisica Internacional, v. 12, p. 55-70.

Negendank, J. F., Emmermann, W. R., Krawczyk, R., Mooser, F., Tobschall, H., y Werle, D., 1985, Geological and geochemical investigations on tile Eastern Trans-Mexican Belt: Geofisica Internacional, v.24, p. 477-575.

Nelson, S.A., y Hegre, J., 1990, Volcan Las Navajas, a PliocenePleistocene trachyte-peralkaline rhyolite volcano in the Northwestern MVB: Bulletiri of Volcanology. 52, p. 186-204.

Nelson S. A. y Hegre J., 1990, Volcan Las Navajas, a PliocenePleistocene trachyte-peralkaline rhyolite volcano in the Northwestem MVB: Bulletin of Volcanology. 52, p. 186-204.

Nieto-Obreg6n, J.; Urrutia-Fucugauchi, J.; Cabral-Cano, E., y Guzmán de la Campa, 1992, Listric faulting and continental rifting in western Mexico-A paleomagnetic and structural study: Tectonophysics, 208, p. 365-376.

Nixon, G.T., Demant, A., Amstrong, R. L., YHarakal, J. E., 1987, K-Ar and geologic data bearing on the age and evolution of the Trans-Mexican Volcanic Belt: Geofísica Internacional, v. 26, p.109-158.

Nixon, G.T., 1989, The geology of the Iztaccíhuatl Volcano and sldjslcentareas of the Sierra Nevada and Valley of Mexico: Geological Society of America Special Paper, 219, 58 p.

Ortega-Gutiérrez, F., Ruiz, J., Centeno-García, E., 1995, Oaxaquia, a Proterozoic microcontinent accreted to North America during tile late Paleozoic: Geology, v. 23, p.1,127-1,130

Pardo, M., y Suárez, G., 1993, Steep subduction geometry of the Rivera plate beneath the Jalisco block in western Mexico: Geophysical Research Letters, 20, p. 2,391-2,394.

Pardo, M., y Suárez, G., 1995, Shape of the subductad Rivera and Cocos plates in southern Mexico: seismic and tectonic implicationa: Joumal of Geophysical Research, 100, p. 12,35712,373 .

Pasquare, G. (direction); Ferrari, L. (con1pilation) et al., 1991, Geological map of tile central sector of Mexican Volcanic Belt, States of Guanajuato and Michoacan. Geological Society of America Map and Chart series, MCH 72. Pasquare G. (direction), Ferrari L. (compilation) et al., 1991. Geological map of tile central sector of Mexican Volcanic Belt, States of Guanajuato and Michoacán: Geol. Soc. Am. Map and Chart series, MCH 72.

Pérez-Venzor, JA; Aranda-Gómez, J.J.; McDowell, F.w., y SolorioMungufa, J.G., 1997, Geología del Volcán Palo Huérfano, Guanajuato, México: Revista Mexicana de Ciencias Geológicas, vol. 13, no. 2, p. 174-183.

Pradal, E., y Robin, C., 1994, Long-lived magmatic phases at Los Azufres volcanic center, Mexico: Journal of Volcanology and Geotilermal Research, 63, p. 201-215.

Righter, K., y Carmichael, I.S.E., 1992, Hawaiites and relatad lavas in the Atenguillo graben, western Mexican Volcanic Belt: Geological Society of America Bulletin, 104, 1,592-1,607.

Righter, K.; Carmichaei, I.S.E., y Becker, T., 1995, PlioceneQuaternary volcanism and faulting at the intersection of the
Gulf of California and the Mexican Volcanic Belt: Geological Society of America Bulletin, 107, p. 612-626.

Robin, C., y Boudal, C., 1987, A gigantic Bezymianny-type event at the beginning of modern volean Popocatépetl: Journal of Volcanology and Geothermal Research, v. 31, p. 115-130.

Rodríguez-Elizarrarás, S., 1995, Estratigrafía y estructura del volcán de Colima, México: Revista Mexicana de Ciencias Geológicas, 12, p. 22-46.

Rosas-Elguera, J.; Ferrari, L.; Garduño-Monroy', V.H., y UrrutiaFucugauchi, J.,1996, Continental boundaries of the Jalisco block and their influence in the Pliocene-Quaternary kinematics 01 westem Mexico: Geology, 24, 10, p. 921-924.

Rosas-Elguera, J.; Ferrari, L.; López-Martínez, M., y UrrutiaFucugauchi, J., 1997, Stratigraphy and Tectonics of the Guadalajara Region and Triple Junction Area, Westem Mexico: International Geology Review, 39, p. 125-140.

Siebe, C.; Komorowski, J.C., y Sheridan, M.F., 1992, Morphology and emplacament of an unusual debris-avalanche deposit at jocotitlan volcano, central Mexico: Bulletin of Volcanology, 54, p.573-589.

Siebe, C., Abrams, M., Macias, J.L., Obenholzner, J., 1996, Repeatad volcanic disasters in Prehispanic time at Popocatepetl, central Mexico; pást key to tile future?: Geology, 24, p. 399-402

Stoopes, Gary R., y Sheridan, Michael F., 1992, Giant debris avalanches from the Colima Volcanic Complex, MexicoImplications for long-runout landslides (>100 km) and hazard assessment: Geology, 20, p. 299-302.

Suárez, G.; García-Acosta, V., y Gaulon, R., 1994, Active crustal deformation in the Jalisco block, Mexico-evidence for a great historical earthquake in the 16th century: Tectonophysics, 234, p.117-127.

Suter, M.; Quintero, O., y Johnson, CA, 1992, Active faults and state of stress in the central part of the Mexican Volcanic Bel-the Venta de Bravo fault: Journal of Geophysical Research, 97, p. 11,983-11,994.

Suter, M.; Quintero, O.; López, M.; Aguirre, G., y Farrar, E., 1995a, The Acambay graben-active intra-arc extension in the TransMexican Volcanic Belt, Mexico: Tectonics, 14, p. 1,245-1,262.

Suter, M.; Carrillo, M.; López, M., y Farrar, E., 1995b, The Aljibes halfgraben, active extension at the boundary between the transMexican Volcanic Belt and the southern Basin and Range: Geological Society of America Bulletin, 107, p. 627-641.

Urrutia-Fucugauchi, J., y Flores-Ruiz, J.H., 1996, Bouguer gravity anomalies and regional crustal structure in central Mexico: Intemational Geology Review, vol. 38, p. 176-194.

Valdéz-Moreno, G. y Aguirre-Díaz, G.J., 1996, Volcán La Joya: Un ejemplo de magmatismo inicial del Cinturón Volcánico Mexicano: VI Congreso Nacional de Geoquímica. Actas INAGEQ, vol. 2, p. 51-56.

- Verma, S.P., y Nelson, S.A., 1989, Isotopic and trace element constraints on the origin and evolution of alkaline and calcalkaline magmas in the northwestern Mexican Volcanic 8elt: Joumal of Geophysical Research, v. 94, p. 4,531-4,544.

Wallace, P.; Carmichael, I.S.E.; Righter, K., y Becker, T., 1992, Volcanism and tectonism in western Mexico: A contrast of style and substance: Geology, 20, p. 625-628.

Wallace, P., y Carmichael, I.S.E., 1999, Quaternary volcanism near the Valley of Mexico: implications for subduction zone magmatism and the effects of crustal thickness variations on primitive magma compositions: Contributions to Mineralogy and Petrology, v. 135, p. 291-314. 